\title{
Importance of Carbon Contamination in High-Resolution (FEG) EPMA of Silicate Minerals
}

\author{
Ben Buse, Stuart Kearns
}

School of Earth Sciences, University of Bristol, Bristol, UK

High-resolution electron probe microanalysis (EPMA) of silicate minerals is now possible with fieldemission gun (FEG) instruments. Applications include studying volcanic processes on short time scales through diffusion studies [e.g. 1] and analyzing high-pressure experimental run products and partition coefficients with often sub-micron sized phases.

To achieve high-resolution analysis a low accelerating voltage must be used providing a small analytical volume together with a small beam size as provided by the FEG. Carbon contamination (carbon deposition as a result of thermal cracking of vapour phase hydrocarbons under the beam) is important at these conditions as the accumulating carbon reduces the energy of the beam electrons as they pass through it [2]. At low over-voltages even a small reduction in the energy of beam electrons reaching the sample results in a significant drop in X-ray excitation [2]. Contamination does not form a uniform film of carbon but forms a ring shaped deposit with most of the carbon deposited around the point of beam impact (Figure 1a). This is a problem for high-resolution multiple point lines where successive points sit on the carbon ring of the previous point $[3,4]$.

Here, contamination is examined on carbon-coated silicate minerals at $5 \mathrm{kV}$ and $10 \mathrm{nA}$. For individual spot analyses the contamination has no effect on X-ray intensities with all the carbon deposited in a ring adjacent to the analysis position. Using BSE calibrated images the rate of carbon buildup within these rings was measured; the use of a cold trap dramatically reduces the amount of carbon deposited (Figure 1a, b) both in terms of the rate of deposition and the amount at which carbon deposition levels off within 1 minute.

For multipoint lines the first point is unaffected but all subsequent points partially overlay the carbon ring of the previous point. This is recorded by a rise in measured carbon and a concomitant drop in other $\mathrm{X}$-ray intensities. This can be minimized by using a cold trap with losses observed in X-ray intensity for $\mathrm{Si}$ of $1-2 \%$ relative and $\mathrm{Ca}$ of $3-4 \%$ relative at a $30 \mathrm{~nm}$ beam diameter with a $0.4 \mu \mathrm{m}$ spacing on a homogenous andradite. Further analytical improvements are possible by using the measured rise in carbon to correct for the loss in X-ray intensity. Andradite and Cr-diopside samples with different thicknesses of carbon coat were used to derive an experimental correction for the loss of X-ray intensity with carbon thickness expressed in k-raw (unknown intensities/standard intensities). This correction was then used to correct a series of multipoint lines in different silicate minerals (andradite, Mg-olivine and Fe-olivine). Using the experimental correction, errors of ca. 3\% were achieved for multipoint lines of $400 \mathrm{~nm}$ spacing and $30 \mathrm{~nm}$ beam diameter without using a nitrogen cold trap (Figure 2). By using both the correction and a cold trap, errors of $<1.5 \%$ can be achieved (Figure 2). The experimental correction was compared to empirical corrections based on data calculated using GMRFilm [5].

The experimental correction gave the best results although a GMRFilm based correction using the Bastin 1990 matrix correction [6] also gave reasonable results except for $\mathrm{Ca}$ which at $5 \mathrm{kV}$ has a very low overvoltage. 


\section{References:}

[1] Saunders et al, Chemical Geology 364 (2014), p. 20

[2] SJB Reed in "Electron Microprobe Analysis" , (Cambridge University Press, Cambridge)

[3] GF Bastin and HJM Heijligers in "Quantitative electron probe microanalysis of carbon in binary carbides” , Technical Report, (Eindhoven University of Technology, Eindhoven, Netherlands)

[4] PT Pinard et al, Microscopy and Microanalysis 19 (2013), p. 996.

[5] RA Waldo, Microbeam Analysis 23 (1988), p.310.

[6] GF Bastin and HJM Heijligers, Scanning 12 (1990), p. 225

a)
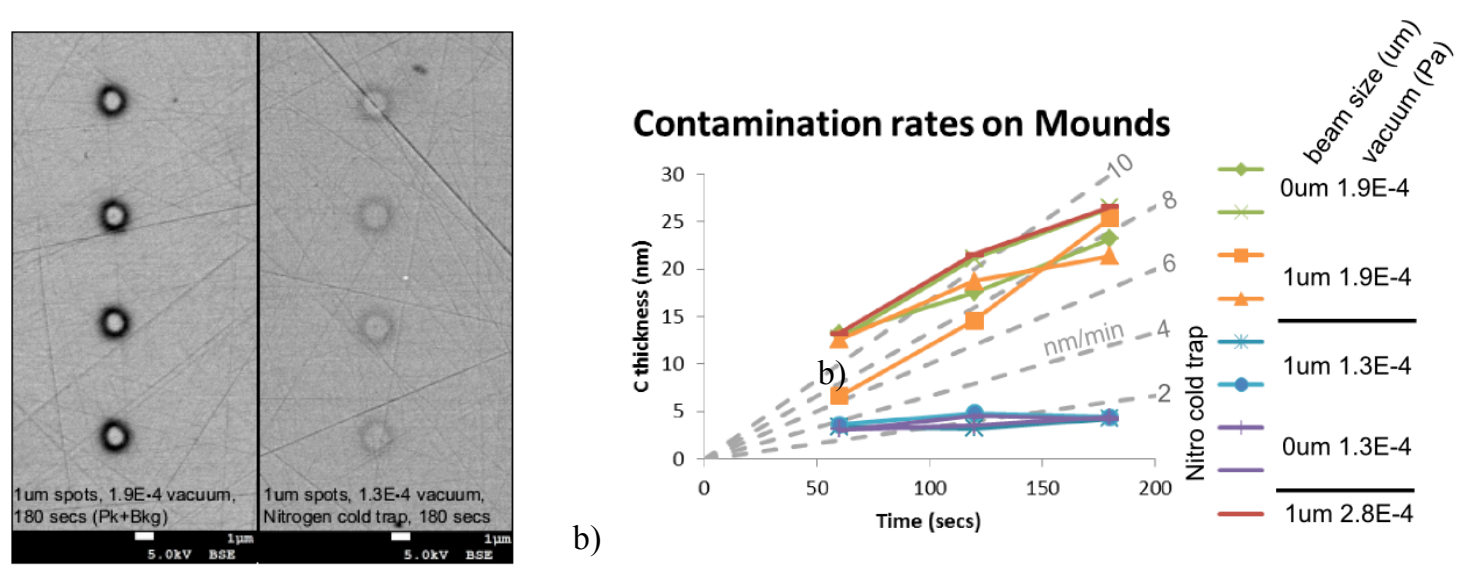

Figure 1. (a) BSE images of carbon contamination rings around analysis spots without (left) and with using a nitrogen cold trap (right). (b) Amount of carbon deposition within the adjacent rings for different analytical times as measured from calibrated BSE images. Analytical conditions $1 \mu \mathrm{m}$ beam diameter, $5 \mathrm{kV}$ and $10 \mathrm{nA}$ on andradite.
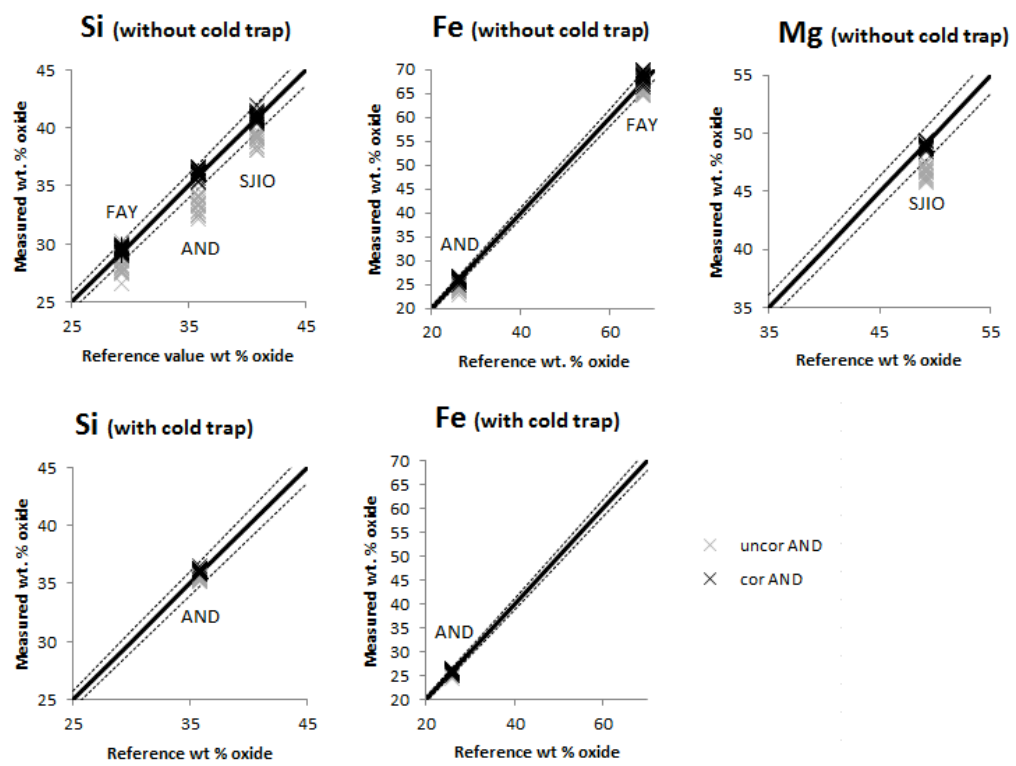

Figure 2. Multipoint line measurements from andradite (AND), fayalite (FAY) and St John's Island olivine (SJIO). In black is data corrected for contamination using an experimentally derived empirical correction. Data is for lines run with and without a nitrogen cold trap. Dashed lines are $+/-3 \%$ relative from reference values. 